\title{
Structural Health Monitoring: Alarming System
}

\author{
Adel ElSafty, Ahmed Gamal, Patrick Kreidl, Gerald Merckel \\ School of Engineering, University of North Florida, Jacksonville, USA \\ Email: adel.el-safty@unf.edu, ahmed.gamal@unf.edu,patrick.kreidl@unf.edu,gmerckel@unf.edu
}

Received April 7, 2013; revised May 7, 2013; accepted May 22, 2013

Copyright (C) 2013 Adel ElSafty et al. This is an open access article distributed under the Creative Commons Attribution License, which permits unrestricted use, distribution, and reproduction in any medium, provided the original work is properly cited.

\begin{abstract}
There is a huge investment in our infrastructure that is vital to our social and economic life. However, the aging and deterioration of the structures require implementing a damage detection system to monitor their structural integrity. In this study, a new alarming system was developed as part of a structural health monitoring system and installed in a scaled-down structure models. The designed system incorporated microprocessors, wireless communication, transducer, and cellular transmission that allow remote monitoring. The developed system facilitates continuous monitoring process of any part of structures and controlled remotely from any location. The system was equipped with data processing subsystem that detects structural behavior irregularity, defects, and potential failures. The system was tested using Linear Variable Differential Transformer (LVDT) for deflections and using strain gages to measure the developed axial and flexural strains in different structural models. Filtering algorithm was used to filter graphs and the data gathered in each loading stage was averaged and plotted to show the abrupt change in the values. The filtering system helps the alarming system to have a clear prediction of possible irregularities. The developed system provides the desired features of low cost, low power, small size, flexibility and easy implementation, remote accessing, early detection of problems, and simplified representation of the results.
\end{abstract}

Keywords: Monitoring; Wireless; Cellular; Data Processing

\section{Introduction}

In the United States, $50 \%$ of all bridges were built before 1940 s, and approximately $42 \%$ of these structures are structurally deficient [1]. There are a limited number of bridge inspectors available to inspect the nearly 600,000 bridges in the US once every 2 years [2,3]. The manual inspection measurements are subjected to human error and their interpretation relies on the inspectors' judgment. Therefore, there is a tremendous need to develop new techniques of structural health measuring.

SHM is used for rapid condition screening and for providing reliable information regarding the integrity of the structure, in near real time [4]. The sensor systems that need to be developed should have the potential for wireless, in-situ, and distributed sensing that provides near real time continuous monitoring. This will help providing timely warning to mitigate any potential failure and identify any damage before further progressing. New structural health monitoring systems have been developed. Some systems use wireless sensors with self-diagnosing and self-calibration to reduce data transmission, thus reducing power consumption [5]. Other systems implemented wireless sensors that are capable of actua- tion, in which the sensor node is able to fix the detected problems without sending the data to the control node so that the used power can be reduced [6]. There was also a different approach based on covering the structures with piezoelectric layer and sending mechanical impedance through the layer and then measuring the electrical impedance of the layer [7]. Many researchers tried to overcome the problem of power consumption by different techniques such as solar and wind energy, self-acquisition of sensors, and sensors actuation. Researchers also investigated reducing the size of the system, increasing the read range, providing more memory, and improving the ruggedness and robustness of the sensor node.

Previous studies indicate that excessive strain, excessive vibration, excessive deflection, corrosion, temperature changes, or fatigue are considered among the most factors affecting structures' health. Any abnormal increase in any of the measured parameters including strains and stresses or initiation of cracks in the structures should be detected. There is a need for new structures health monitoring systems to be developed utilizing the advances in the microprocessors and communication technologies. The new system should have the following 
specifications: low cost and low energy consumption, remote accessing, high speed communication, real time identification of the problems, simplified representation of the results, disaster patterns for early identifications of problem, less space, and easy implementation.

The developed monitoring system is designed to monitor the main parameters affecting the health of bridges in particular. Through observation of the system over time using continuously recorded response measurements from sensors and the extraction of damagesensitive parameters from these measurements to determine the near real-time state of structure's health. An estimate of the overall health of a system can be reached through looking for changes in parameters that are sensitive to the change [8]. The new system developed in this study is able to monitor strain, deflection, temperature, and corrosion.

\section{Experimental Setup}

The experiment was aimed to develop a new system having remote accessing and capable of early identification of problems facing the structure. The experimental setup was divided into three stages for better development. The three stages of the experimental setup are:

1) Sensor node

2) Transmission

3) Data processing and presentation

\subsection{Sensor Node}

In this stage the size of the sensor node is required to be reduced which allows easier implementation of the node in any location of the structure. In this stage, electrical engineering and civil engineering concepts are integrated in order to achieve the desired outcome. The system utilizes the advanced technology in microprocessor. In this sensor node, the transducer was attached to the microprocessor through interface circuit equipped with the necessary functions blocks within less size while increasing the accuracy of the output. The interface circuit aims to collect and amplify the output from the transducer and feed this output to the microprocessor. The analog-to-digital convertor (ADC) on the microprocessor converts the transducer readings to digital readings in order to be handled by the microprocessor. The interface circuit contains a differential circuit followed by an amplification circuit, Figure 1 [9].

The differential circuit is represented by a differential amplifier that has a gain, Equation (1), equals to

$$
G 1=\frac{R 5}{R 4}=\frac{R 6}{R 3}
$$

while the amplifying circuit is composed of a noninverting amplifier with gain, Equation (2), equals to

$$
G 2=\frac{R 1+R 2}{R 2}
$$

the output from the amplification circuit is fed to the input pin of the ADC which converts the analog input to digital output through the following equation, Equation (3). The output from the ADC is stored in the memory registers located on the microprocessor board to be processed and transmitted.

$$
N=\frac{2^{12} \times V_{\text {in }}}{2.5}
$$

each sensor node has a microprocessor (e.g. MSP4 30F6137) representing the central processing unit (CPU), Figure 2, connected to the (ADC). The readings from the transducer go through the conversion cycle while it is compared to the predefined safety values through the comparator found in the CPU. When the input to the system is approaching or exceeding this safety values, the alarming subsystem on the sensor node is activated. The alarming system light up the safety light for instant identification of the irregularity for any nearby assistance. The alarming system activates the RF antenna located on the microprocessor board immediately. The antenna receives an interrupt (alarming message) from the CPU to send a warning alarm to the main control unit for immediate action. Figure 3 shows the alarming system safety light. Figure 3(a) shows the safety light turned with no load added to the system, while Figure 3(b) shows the safety light being lit up due to exceeding the safety value.

To test the sensor nod subsystem of the system, two different setups were constructed to implement the sensor nodes. The first structure was composed of a steel plate supported on two wooden blocks which was used as preliminary setup to test strain gages and Linear Variable Differential Transformer (LVDT) through flexural bending under three-point loading, Figure 4. The second setup was a small-scale bridge composed of interconnected truss members. The truss bridge was loaded with stationary and moving loads, Figure 5. This bridge setup was used to verify the preliminary readings gathered from the first setup of steel plate structure through bending of the

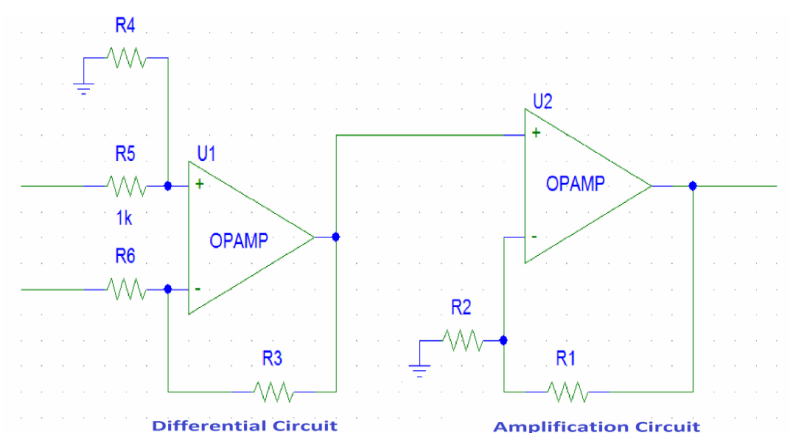

Figure 1. Interface circuit schematic. 


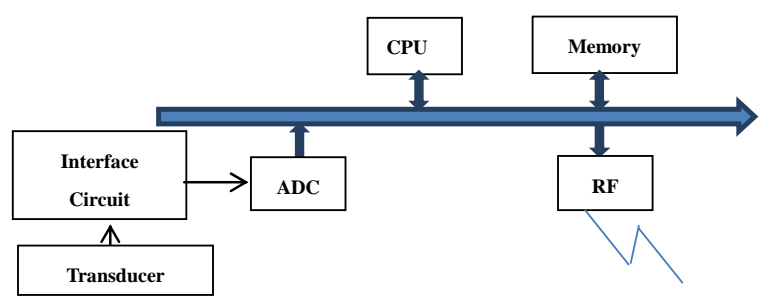

Figure 2. Typical sensor diagram.

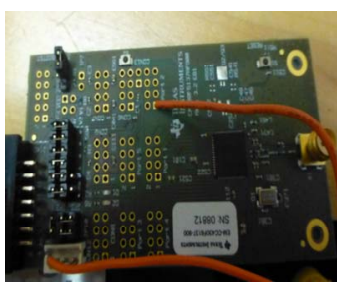

(a)

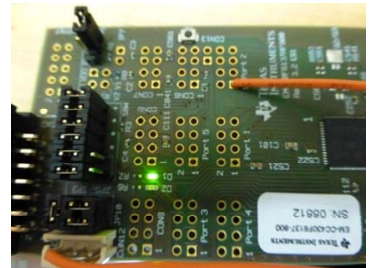

(b)
Figure 3. Safety lights. (a) Safety light off; (b) Safety light on.

bridge track/deck as well as testing the axial strains developed in the truss bridge members.

\subsection{Transmission}

The transmission of data is done after the data is collected through the sensor node. The system included three subsystems, as shown in Figure 6. The first subsystem was composed of sensor nodes that were connected to several members of the structure. The second subsystem was the main control unit. It was connected to the sensor nodes subsystems through wireless communication. The third subsystem was the monitor unit that represents the final stage for the data and was connected to the main control unit through cellular data transmission.

The control unit is considered to be the brain of the entire system and it acts as a link between the measuring device and the end user. The second subsystem (main control unit) consists of a microprocessor (e.g. MSP430) connected to an RF transmission unit and cellular transmission unit. The control unit is the intermediate stage responsible for conveying data from the sensors node to the monitor unit. It also served in carrying commands from and into the sensors node and from and into the monitor unit. To save power, the control unit stays in a low power mode until it is activated by the sensor node or the monitor unit. When the control unit is activated, the cellular transmission unit and RF transmission unit are activated to receive data from the sensor node and send it to the monitor unit, or to receive commands from the monitor unit and send them to the sensor node.

The monitor unit is the user interface that represents the final destination of the collected data. The function of the monitor unit is to receive commands from the user

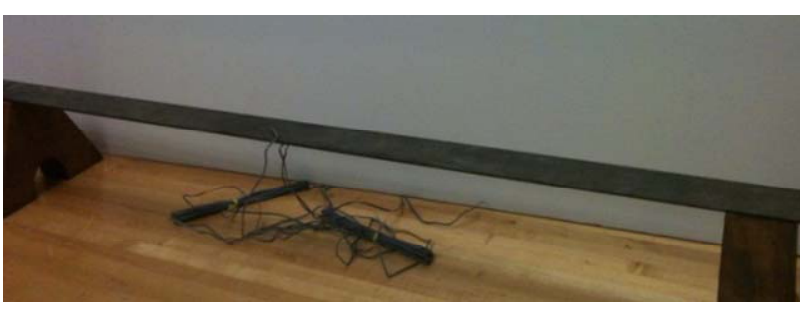

Figure 4. Steel bar setup.

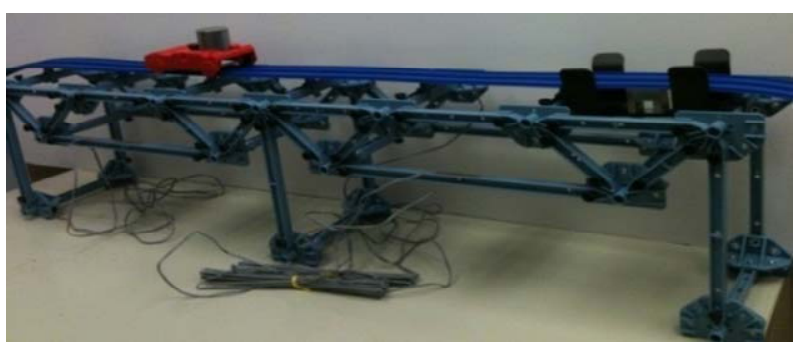

Figure 5. Bridge setup.

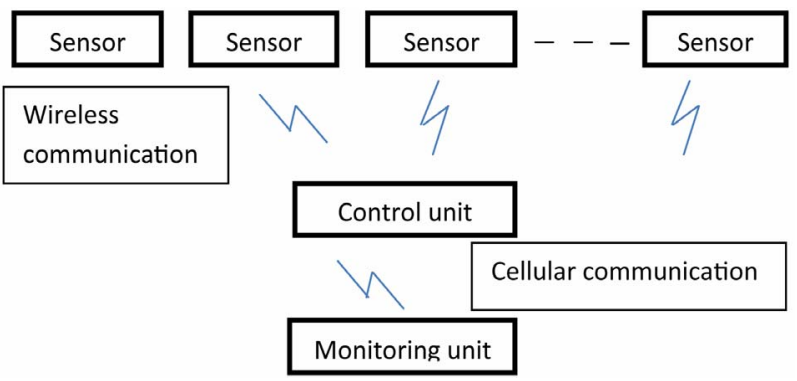

Figure 6. System design.

and send them to the control unit as well as receiving data from the control unit. The monitor unit has software development kit. The software is web-based program responsible for collecting, processing, and displaying the data in a graphical representation. Moreover, the software alerts the user when any exception or irregularity takes place in the data collected.

A communication protocol was defined to ensure a proper secured transmission of the correct messages. This protocol is recognized by both the sending and the receiving ends and it represents the conversation between the two ends to verify the complete transmission of the correct message. The sending end collects necessary information about the message to be sent and the message is sent with its information to the transmitting end of the RF transmission channel, thus starting communication. The transmitting end sends a sending acknowledgment to the receiving end. When the receiving end receives the acknowledgement, the transmitting end starts to send the information about the message followed by the message itself. Once the complete message is received, Cyclic Redundancy Check (CRC) register checks the message received to verify that the correct message is received. After verifying receiving the correct message, an ac- 
knowledgement message is sent back to the transmitting end. When a wrong or incomplete message is sent or no acknowledgement is received from the other end, the transmission end restarts the transmission of the data. This step is repeated for three times. If the same situation continues after the third time, the transmitting end sends an error report to the CPU which calls for immediate action. Figure 7 shows the transmission protocol flow chart.

The structure of the message being sent between the transmitting end and the receiving end is represented by a packet structure, as shown in Figure 8. The message is divided into four parts. Each part of the message is a multiple of a byte long and carries certain information about the message.

Each part of the message is defined as follows:

1) Sender and Receiver (S/R) Address: 1 Byte indicates the address of the sender node and the receiving node with the most significant bit (MSB) reserved for address extension for more nodes to be connected. Example of node address are:

0001: Control unit. 0010: Sensor 1/unit 1 .

0011: Sensor 2/unit 1. 0100: Sensor 3/unit 1 .

2) Identity byte: 1 Byte contains the necessary information to identify the transmitted message. It includes 1 bit for priority (error), 1 bit as initiator to indicate the presence of data message attached to the message being sent, and packet size. In case an error is being sent, no data can be sent at the same time. Table 1 shows an iden- tity byte example.

3) Command/Error

a) Error byte: The error byte indicates the presence of an error in any part of the system, as shown in Figure 9. Each bit in the error byte refers to a certain part of the system; the high state of this bit indicates the presence of an error.

b) Command byte: The command byte is always preceding the data bytes to identify the type of the data sent and any command need to be sent to the receiving end. The first four bits identify the type of data sent. Each measuring device is assigned one bit, and the high state of the bit indicates the data transmission from this device. If more than one device is activated at the same time, the data are transmitted according to the sequence of the devices, as follows: strain gages, LVDT, temperature sensor, and corrosion. The last four bits are reserved for the commands to be sent to the receiving end, as shown in Figure 10.

4) Data message: $X$ bytes indicating the data message transmitted. The number of bytes of the message is identified from the packet size transmitted earlier. Each device is assigned one byte of data.

\subsection{Data processing and presentation}

In the monitor unit, the data go through data processing to convert the data to its original format and then to its parameter equivalence to reach the final format of the

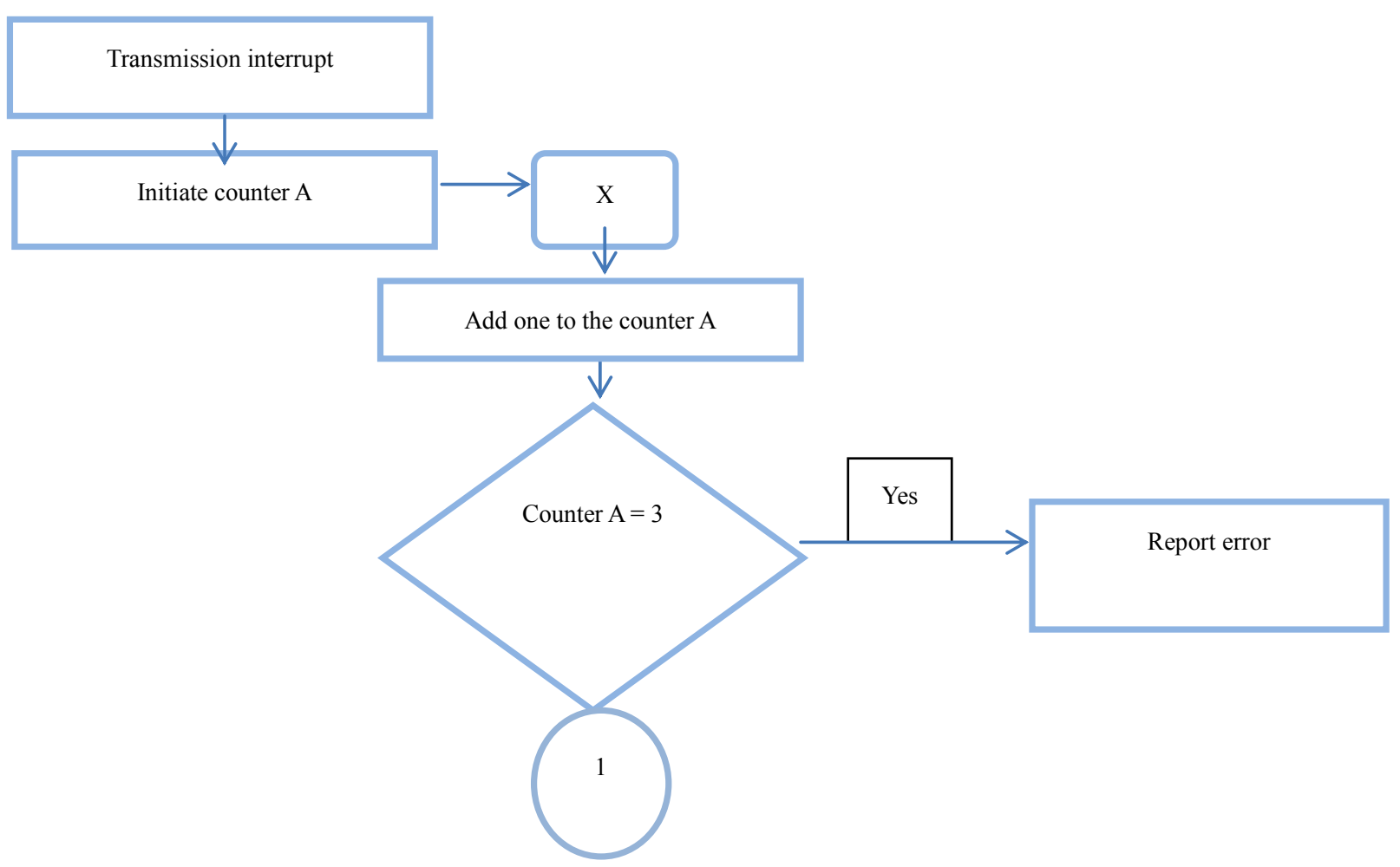

(a) 


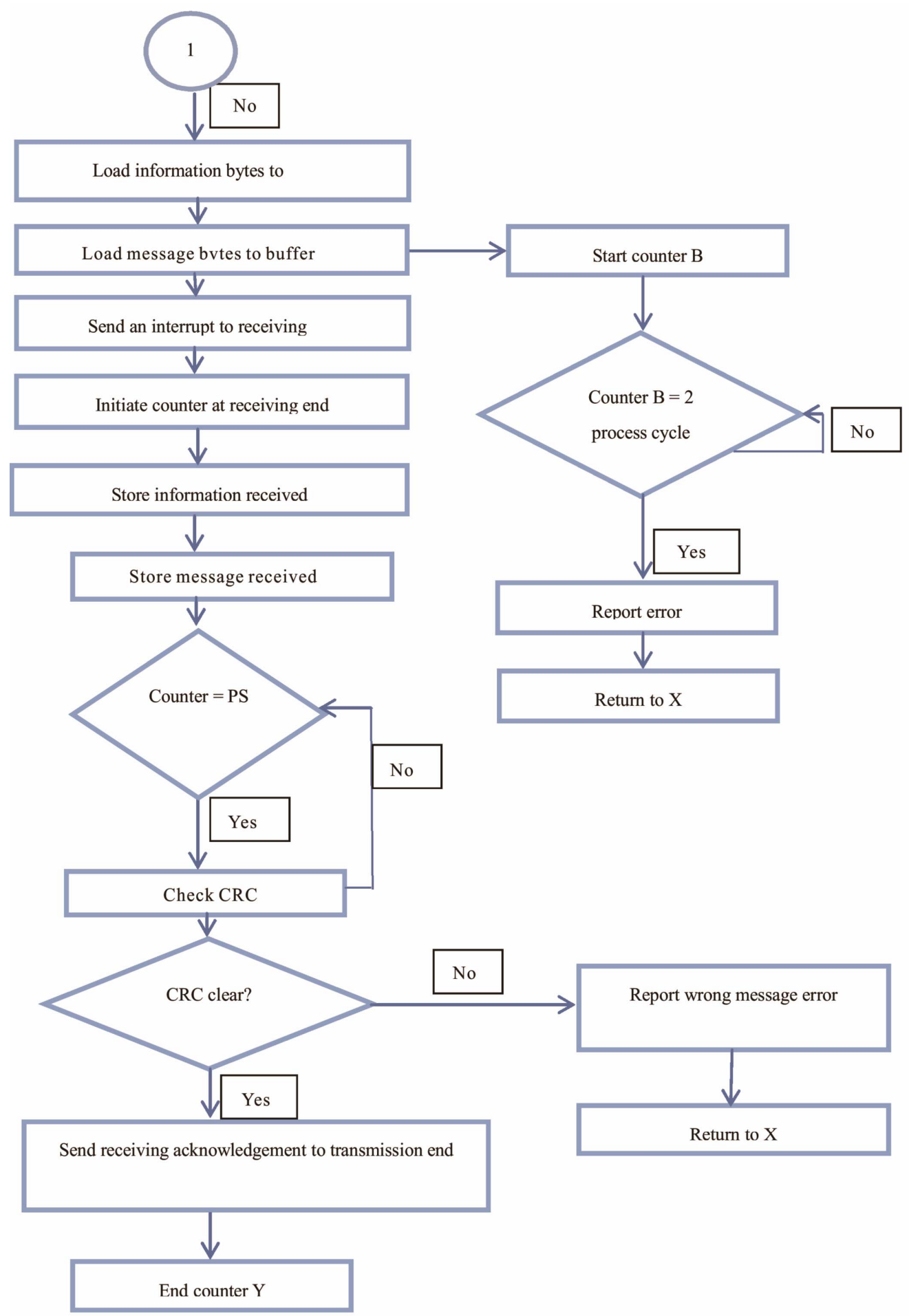

(b)

Figure 7. Transmission protocol flowcharts.

readings which can be presented to the end user. Firstly, the digital data is converted back to its analog format using Digital-to-Analog Convertor (DAC) located on the microprocessor board. The conversion is followed by voltage conversion cycles to convert the data to its de- sired parameters equivalence. After reaching the final format of the readings, they are presented for review using graphical representations and table forms for easier interpretation. Those presentations are stored into a webbased database through MySQL or any similar soft- 


\begin{tabular}{|l|c|c|c|}
\hline $\begin{array}{c}\text { S/R } \\
\text { address }\end{array}$ & $\begin{array}{c}\text { Identity } \\
\text { byte }\end{array}$ & $\begin{array}{c}\text { Command } \\
\text { / error }\end{array}$ & Data \\
\hline 1 byte & 1 byte & 1 byte & X byte \\
\hline
\end{tabular}

Figure 8. Packet structure.

ware. Data processing and data presentation can be conducted using MATLAB or any similar software.

\section{Results and Discussion}

The system was assembled to be tested. In this testing, strain gages are used as transducers to measure strain. The concept of Wheatstone bridge was used for strain gages connection. In this bridge connection, the strain gage replaces one of the resistors in the bridge in case of using quarter bridge or two strain gages replaces two resistors in the bridge in different directions in case of using half bridge, Figure 11.

The interface circuit was assembled to provide the required amplification using the following resistors' values in order to have output values in a required range for safe processing and transmission, $\mathrm{R} 5=\mathrm{R} 6=120 \mathrm{k} \Omega, \mathrm{R} 7=$ $\mathrm{R} 8=1 \mathrm{M} \Omega$, while $\mathrm{R} 9=20 \mathrm{~K} \Omega$ and $\mathrm{R} 10=1 \Omega$. The gain was calculated as shown in Equations (4)-(6):

$$
\begin{aligned}
& G 1=\frac{1 \mathrm{M}}{100 \mathrm{~K}}=10 \\
& G 2=\frac{20 \mathrm{~K}+1 \mathrm{~K}}{1 \mathrm{~K}}=20 \\
& G=G 1 \times G 2=200
\end{aligned}
$$

the entire circuit pictures and schematic are shown in Figures 12-15.

Since assessment of damage usually require a comparison between two system states, several strain measurements were recorded at different load levels. To test the efficiency of the sensor node, three tests were conducted. In the first test, the strain gages were attached to the steel plate top and bottom surfaces at mid span. The gages measured the developed bending strains in the steel plate that was subjected to 3-point loading. This test is a static flexural testing. The second test was conducted through attaching the strain gages to the track of the truss bridge to measure the bending strains developed in the track/deck due to the movement of a truck loaded with different weights. This test is a dynamic testing. The third test was conducted on the truss bridge and measures the tension strains developed in the bridge members due to applying loads to the bridge. This test is a static testing. The voltage output from the circuit was recorded by the ADC where it is converted to digital values and transmitted

\begin{tabular}{|c|c|}
\hline 10000010 & High priority message. An error occurred \\
\hline $01 \mathrm{xxxxxx}$ & Data message is attached \\
\hline 01000010 & $\begin{array}{c}\text { Data message of size } 3 \text { bytes. } 1 \text { data byte } \\
\text { attached }\end{array}$ \\
\hline $11 \mathrm{xxxxxx}$ & Unidentified byte structure \\
\hline
\end{tabular}
through RF antenna. The digital values were converted
Table 1. Identity byte examples.

back to their original values at the receiving end. The following equations, Equations (7) and (8), were used to convert the voltage values to its equivalent strain values [10]:

For quarter bridge connection:

$$
\varepsilon=-\frac{V_{\text {out }} \times 4}{G \cdot F \times\left(V_{\text {ex }} \times 2 V_{\text {out }}\right)}
$$

For half bridge connection:

$$
\varepsilon=-\frac{V_{\text {out }} \times 2}{G \cdot F \times V_{e x}}
$$

where $\varepsilon$ is strain, $V_{e x}$ is the supply voltage to the Wheatstone bridge, and $G \cdot F$ is gage factor. In case of metal strain gages, $G \cdot F$ is equal to 2.019 .

The obtained strain values were stored and presented graphically for better interpretation of the data gathered. To ensure the efficiency of the system, some of the values developed were compared against the values collected from HBM Spider 8 acquisition system through conducting the same procedures. The data conversions and graphical presentation were conducted through GUE-Octave which is equivalent to MATLAB but with open source for demonstration purposes. Some filtering algorithms were used in order to reach smooth representtations of the change in the values of the parameters measured.

\subsection{First Testing Findings}

The first static test was conducted on the steel plate structure; the procedures were conducted several times using half-bridge configuration. The voltage values are recorded as well as the strain values after conversion. The graphs of the strain values versus time were plotted to show change in strain due to applying loads to the plate. The alarming system was tested in this procedure; the safety value was defined as the strain of the biggest load. The alarming system was activated when the strain values was approaching the safety values. The alarming light started flashing when the value was approaching the safety value and lit up when the value exceeded this value. The tables of the values developed were originated. Each table contains the digital values, voltage values, strain values, and nulled/net strain values, as shown in Table 2. The following figures represent the strain values 


\begin{tabular}{|c|c|c|c|c|c|c|c|}
\hline Not used & Strain gages & LVDT & Temp. & Corrosion & Battery & $\begin{array}{c}\text { Wrong } \\
\text { message }\end{array}$ & Transmission \\
\hline
\end{tabular}

Figure 9. Error Byte bits divisions.

\begin{tabular}{|l|c|c|c|c|c|c|c|}
\hline Strain gages & LVDT & Temp. & Corrosion & Command bit & Command bit & Command bit & Command bit \\
\hline
\end{tabular}

Figure 10. Command byte bit divisions.

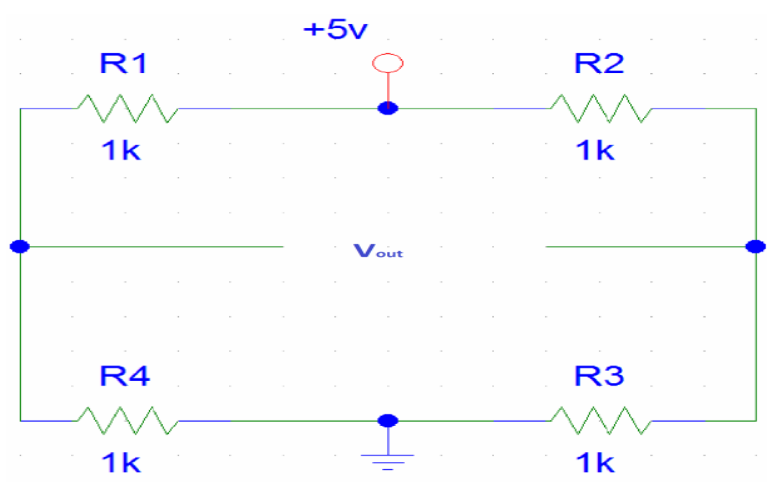

Figure 11. Wheatstone bridge schematic.

associated with applying different loads. Figures 16(a) and (b) represent the change in strain due to applying increasing loads of different weights. Figure 16(c) represents the filtered graph in which every 10 readings are averaged and plotted.

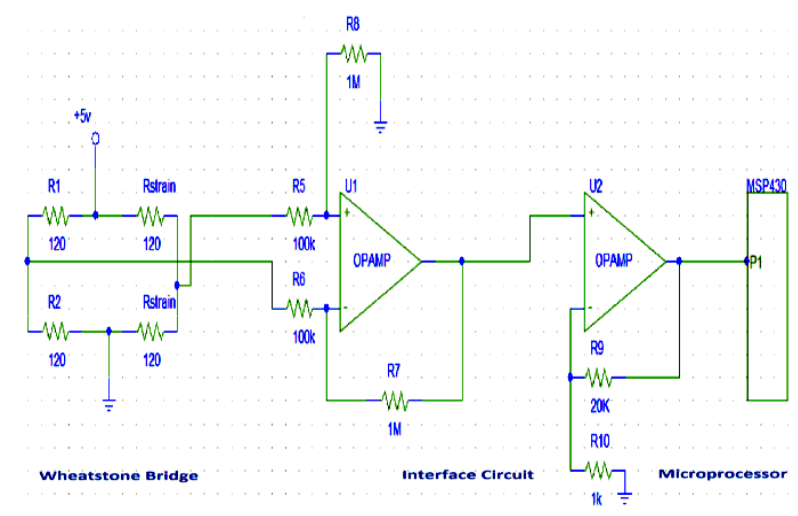

Figure 12. Full system schematic.

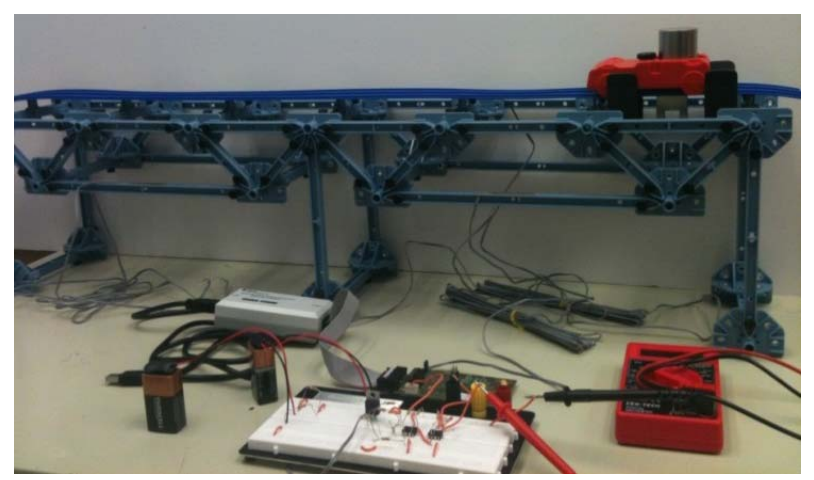

Figure 13. Work station.

\subsection{Second Testing Findings}

The second testing procedure is a dynamic testing conducted on the bridge deck using quarter bridge technique. This test results was used to validate the results gathered from the first testing to increase the credibility of the system. The testing was carried out by moving a loaded truck over the bridge track and measure the bending strain developed in the track/deck. The measuring procedure was repeated several times. Table 3 represents the originated tables as explained in the first testing findings. The following figures represent the recorded strains during moving the loaded truck. Figure 17(a) shows the change in strain when passing the truck twice on the bridge. Figure 17(b) shows the changes when passing the truck three times.

The same procedures in this testing were conducted again by connecting the strain gages directly to HBM Spider 8 acquisition system in order to validate the developed data from the new system. Figure 18 shows the developed graphs from the Spider 8 acquisition system.

\subsection{Third Testing Findings}

This is a static test conducted on the truss bridge member

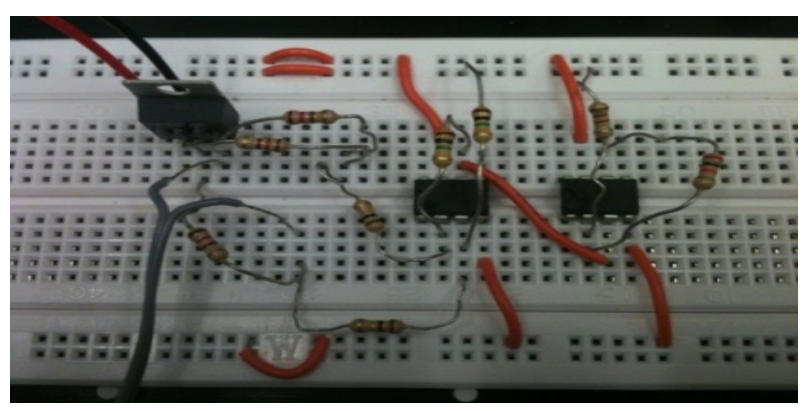

Figure 14. Full system circuit.

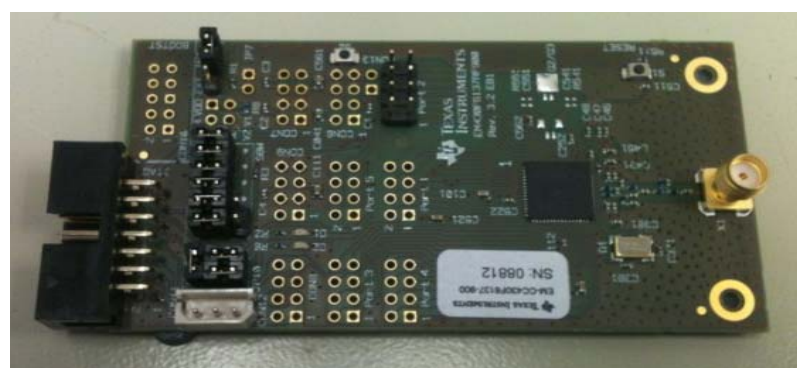

Figure 15. Microprocessor board. 
Table 2. First testing values.

\begin{tabular}{ccc}
\hline Readings & Voltage & Strain Change \\
\hline$[1]$ & 0.014702 & -50.43 \\
{$[2]$} & 0.014702 & -50.43 \\
{$[3]$} & 0.014702 & -50.43 \\
{$[4]$} & 0.014702 & -50.43 \\
{$[5]$} & 0.014702 & -50.43 \\
{$[6]$} & 0.014702 & -50.43 \\
{$[7]$} & 0.013557 & 201.69 \\
{$[8]$} & 0.014702 & -50.43 \\
{$[9]$} & 0.013869 & 132.93 \\
{$[10]$} & 0.014702 & -50.43 \\
\hline
\end{tabular}

Table 3. Second testing values.

\begin{tabular}{ccc}
\hline Readings & Voltage & Strain Change \\
\hline$[1]$ & 0.007918 & -7.51135 \\
{$[2]$} & 0.007959 & -23.6342 \\
{$[3]$} & 0.007951 & -20.4097 \\
{$[4]$} & 0.007874 & 10.22381 \\
{$[5]$} & 0.007906 & -2.67449 \\
{$[6]$} & 0.007922 & -9.12364 \\
{$[7]$} & 0.007951 & -20.4097 \\
{$[8]$} & 0.007874 & 10.22381 \\
{$[9]$} & 0.007906 & -2.67449 \\
{$[10]$} & 0.007898 & 0.55009 \\
\hline
\end{tabular}

with the strain gages in a half bridge configuration. The strain gages are measuring the tensile strains developed in the bridge truss members to validate the efficiency of this system in different application. Table 4 represents some of the recorded data. The following figure represents the change in tensile strain readings by applying different loads to the bridge. Figure 19 shows the increase in tensile strains due to increasing the applied loads and the decrease upon removing the loads.

Using the filtering algorithm, the filtered graphs were developed. In this procedure, the data gathered in each stage of adding different load was averaged and plotted to show the abrupt change in the values. The same procedures were conducted using HBM Spider 8 acquisition system with quarter bridge configuration and Spider $8 \mathrm{~S}$ with half bridge configuration to test the developed

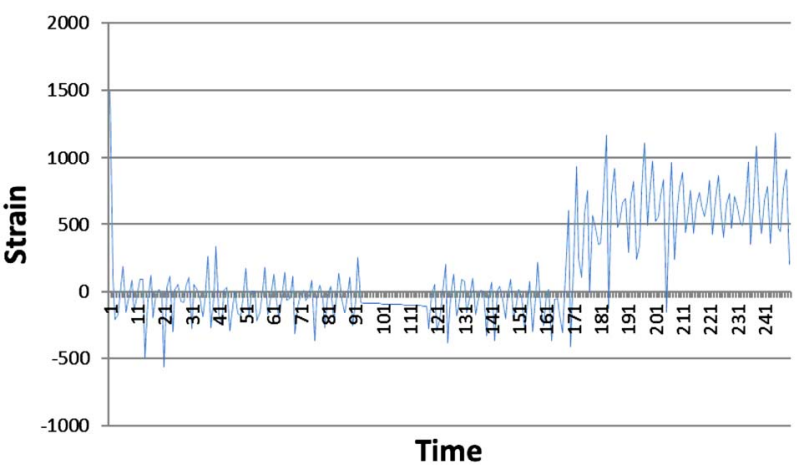

(a)

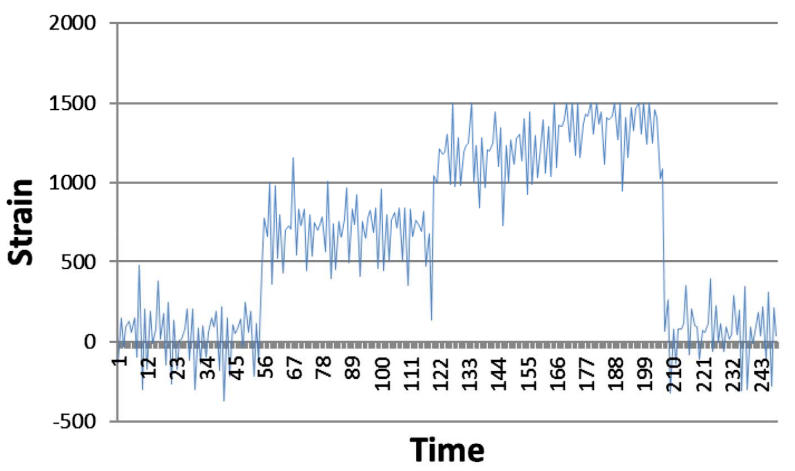

(b)

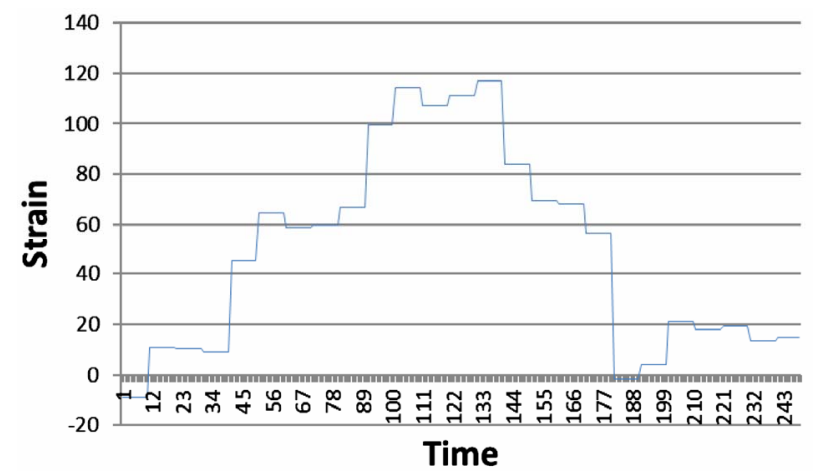

(c)

Figure 16. First Testing strain graphs. (a) Adding two similar loads consecutively; (b) Adding one load towards the end; (c) Filtered strain values representation.

results from the new system. The developed graphs were compared in each configuration of the Wheatstone bridge. The compared graphs showed that all the graphs follow the same trend and the peaks of all the graphs are very close. Figure 20 shows the comparison between the new system filtered graph and Spider 8s graph.

Using the developed system with strain gages to measure strain in different applications validates the efficiency of the system. The results developed show that the generated readings from the system followed the expected trend based on structural analysis concepts. The results ensured that the strains increased when increasing the applied loads. The comparison between the new sys- 


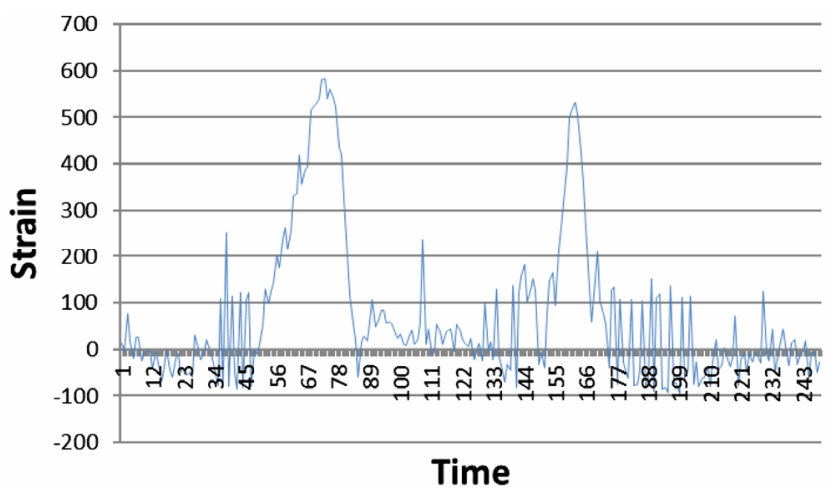

(a)

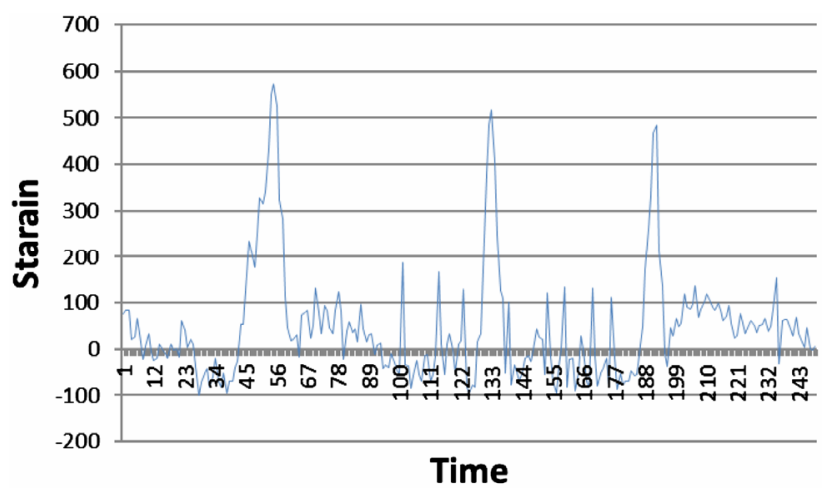

(b)

Figure 17. Second testing strain graphs. (a) Passing the truck twice; (b) Passing the car three times.

$y(t)$ Real-time graph

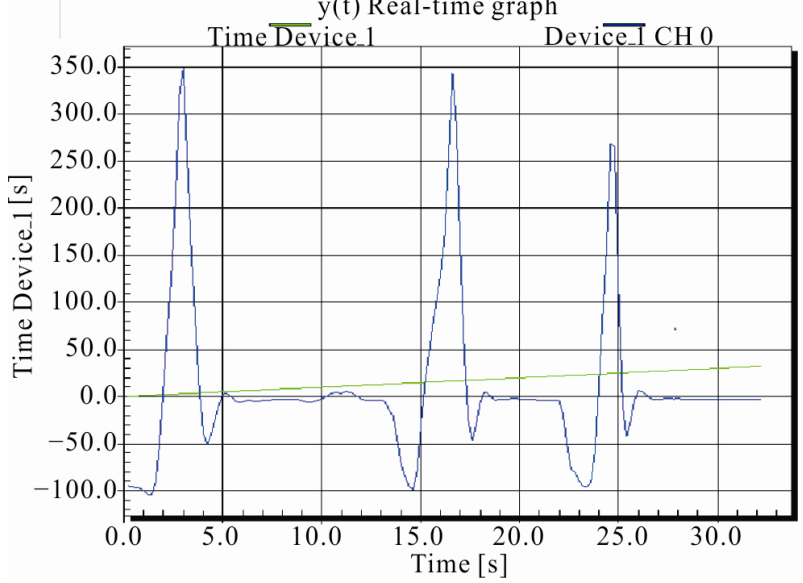

$y(t)$ Real-time graph

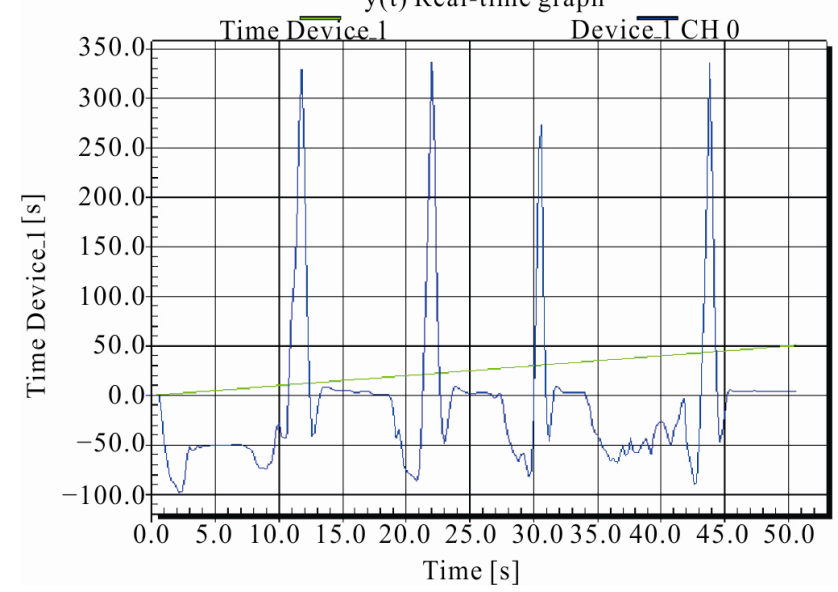

Figure 18. Spider 8 graphs.

tems graphs and the commercial acquisition system graphs shows that the readings fall within the estimated range which proves the reliability of the system. Applying the system with activated alarming system ensures the reliability of the system to early detect the potential disasters affecting the structures. The filtering algorithms enable a better representation of the data gathered for an easier understanding. The filtering system aids the alarming system to have a clear prediction of the possible irregularities.

\section{Conclusions}

Health monitoring of structures is considered as one of the most important concerns when studying structures' performance. An early detection of any irregularity of the structure's performance is the key to an effective monitoring system.

This paper presents the preliminary verification and applicability of wireless monitoring system to monitor the response of bridge structure. The results show that the alarming system can provide broad applications to monitoring and control of civil infrastructures. The
Table 4. Third testing values.

\begin{tabular}{ccc}
\hline Readings & Voltage & Strain Change \\
\hline$[1]$ & 0.013139 & 21.9619 \\
{$[2]$} & 0.01318 & 13.9005 \\
{$[3]$} & 0.013334 & -16.733 \\
{$[4]$} & 0.013228 & 4.2268 \\
{$[5]$} & 0.013033 & 42.9217 \\
{$[6]$} & 0.013326 & -15.1207 \\
{$[7]$} & 0.013367 & -23.1821 \\
{$[8]$} & 0.013167 & 16.3189 \\
{$[9]$} & 0.013147 & 20.3496 \\
{$[10]$} & 0.013025 & 44.534 \\
\hline
\end{tabular}

monitoring involves the observation of the system over time using periodically recorded measurements from an array of sensors. The developed alarming system for 


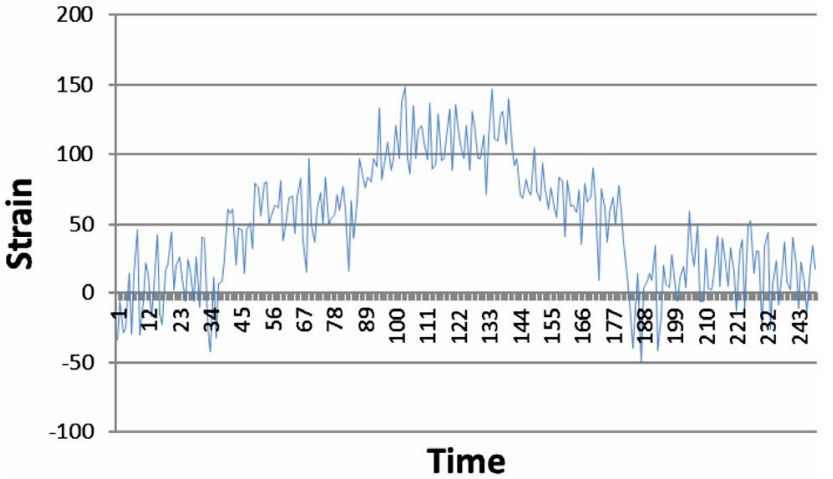

(a)

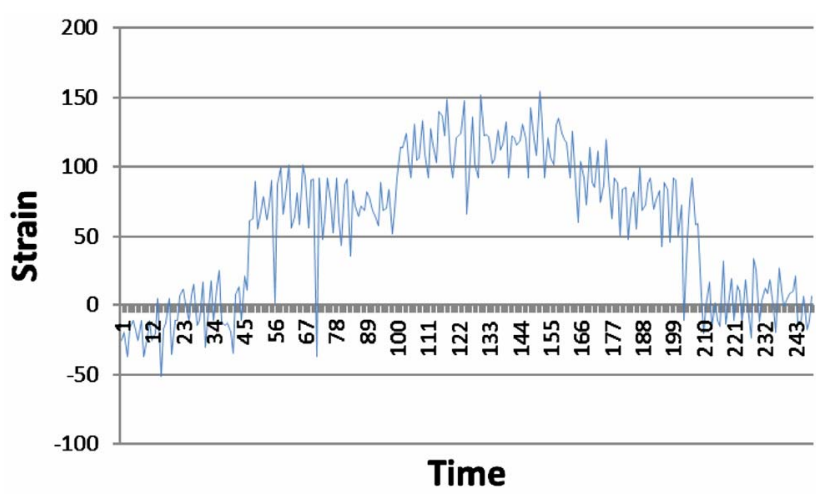

(b)

Figure 19. Third testing strain graphs. (a) Adding two similar loads consecutively; (b) Adding two similar loads consecutively.

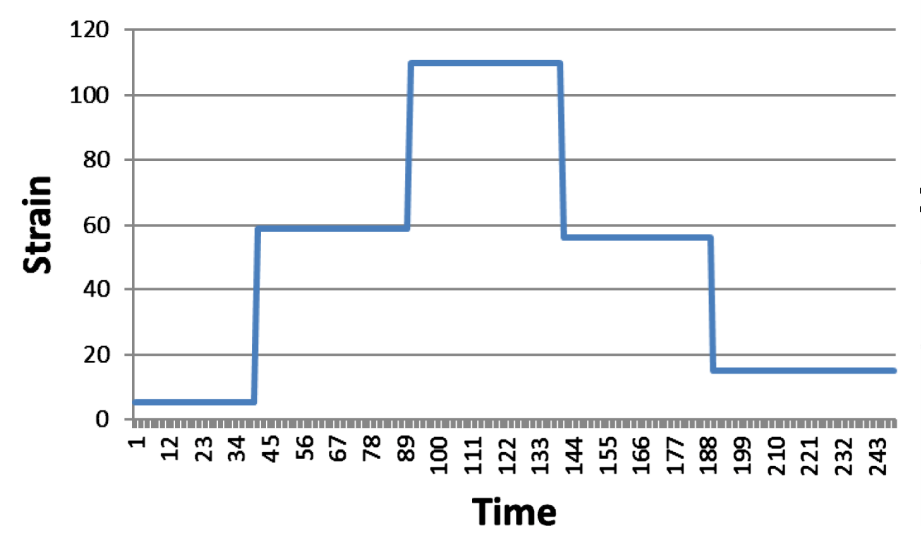

(a)

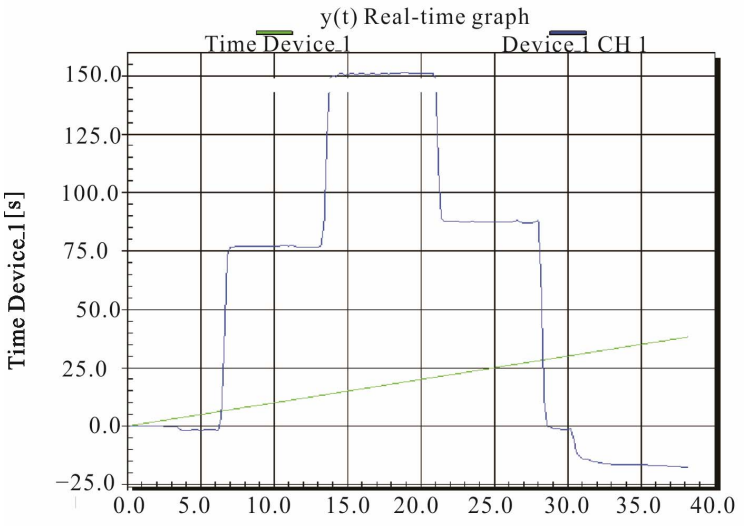

(b)

Figure 20. Data comparison. (a) New System filtered graph; (b) Spider 8S graph.

structural health monitoring serves this purpose. The new system is proved to be able to detect any change in structures performance and activate an immediate alarming system for instant interference. The new systems utilize the advanced technology in wireless transmission and cellular transmission for reliable delivery of the readings. The advanced technology in data mining and data processing facilitate presenting a clear and accurate reading as well as storing sufficient data in a web based database and develop disaster patterns for early identifycation of possible problems. This new system will help to minimize the chances of structural collapses.

The system design and architecture were developed to serve the features of the required system. Using the microprocessor with its on board ADC and memory registers enabled the sensor node to store and process data, this reduces the transmission between the different parts and reduces the power consumed by the system. The cellular transmission allows instant access to the data from any location. The system architecture serves the purpose of secured and reliable transmission. The communication protocol ensures the transmission of a complete and correct message. The packet structure adds the advantage of variable length message of different types such as data message, command message, or error message.

Using the developed simplified electrical circuits to replace the commercially used data acquisition systems will reduce the overall cost. The use of simple electrical circuits connected to the small sized microprocessor board reduced the overall size of the system and facilitate the application of the system in any location. The new system testing showed the efficiency of the tests and its reliability in different applications. The same system can be applied to different transducers with minor modifications. The main idea of the system depends on recording an output voltage from the transducer within a certain range in order to apply it to the microprocessor. The developed system in this study meets all the requirements of lower cost, less power, reduced size, flexibility and easier implementation, remote accessing, early detection of problems, and simplified representation of the results.

\section{REFERENCES}

[1] F. W. Klaiber, K. F. Dunker, T. J. Wipf and W. W. Sanders, "Methods of Strengthening Existing Highway Bridges," NCHRP Report 293, Transportation Research Board, National Research Council, Washington, 1987. 
[2] P. C. Chang, A. Flatau and S. C. Liu, "Review Paper: Health Monitoring of Civil Infrastructure," Structural Health Monitoring, Vol. 2, No. 3, 2003, pp. 257-267. doi:10.1177/1475921703036169

[3] K. P. Chong, J. Nicholas, N. J. Carino and G. Washer, "Health Monitoring of Civil Infrastructures," Smart Materials \& Structures, Vol. 12, No. 3, 2003, pp. 483-493. doi:10.1088/0964-1726/12/3/320

[4] N. Bonessio, G. Lomiento and G. Benzoni, "Damage Identification Procedure for Seismically Isolated Bridges," Structural Control and Health Monitoring, Vol. 19, No. 5, 2012, pp. 565-578. doi:10.1002/stc.448

[5] B. F. Spencer Jr., M. E. Ruiz-Sandoval and N. Krata, "Smart Sensing Technology: Opportunities and Challenges," Journal of Structural Control and Health Monitoring, Vol. 11, No. 4, 2004, pp. 349-368. doi: $10.1002 /$ stc. 48

[6] J. P. Lynch and K. J. Loh, “A Summary Review of Wire- less Sensors and Sensor Networks for Structural Health Monitoring," The Shock and Vibration Digest, Vol. 38, No. 2, 2006, pp. 91-128. doi:10.1177/0583102406061499

[7] D. L. Mascarenas, M. D. Todd, G. Park and C. R. Farrar, "Development of an Impedance Based Wireless Sensor for Structural Health Monitoring," Smart Materials and Structures, Vol. 16, No. 6, 2007, pp. 2137-2145. doi:10.1088/0964-1726/16/6/016

[8] C. R. Bennett, A. Chamorro, C. Chen, H. Solminihac and G. W. Flintsch, "Data Collection Technologies for Road Management," Version-2, East Asia Pacific Transport Unit, The World Bank, Washington, 2007.

[9] A. Gamal, A. ElSafty and G. Merckel, "New System of Structural Health Monitoring," Open Journal of Civil Engineering (OJCE), Vol. 3, No. 1, 2013, pp. 19-28. doi:10.4236/ojce.2013.31004

[10] National Instruments, "Strain Gauge Measurement-A Tutorial,” National Instruments Corporation, 1998. 